\title{
COMPLICACIONES OBSTÉTRICAS DURANTE PUERPERIO MEDIATO, EN RELACIÓN A LA NUEVA CLASIFICACIÓN DE EMBARAZO A TÉRMINO DE LA ACOG, EN EL HOSPITAL DE VITARTE, AÑO 2018.
}

\author{
Eduardo Montenegro Rivera ${ }^{1,3}$, Pedro M. Arango-Ochante ${ }^{2,3}$
}

\begin{abstract}
RESUMEN
Objetivo: Determinar la asociación entre las complicaciones puerperales y la clasificación de Embarazo a Término de la ACOG, en el Hospital de Vitarte, año 2018. Materiales y métodos: El estudio realizado es analítico, retrospectivo de tipo caso y control. La población estudiada estuvo conformada por pacientes con puerperio mediato y que tuvieron alguna complicación durante este lapso. Lo representaron 268 pacientes, con 134 casos y 134 controles con relación de 1:1. La información fue recolectada de las Historias clínicas y estas fueron: edad, grado de instrucción, paridad, $N^{\circ} \mathrm{CPN}, \mathrm{RPM}, \mathrm{IMC}$, hemoglobina, embarazo a término, tipo de parto, duración de parto vaginal, uso de oxitócicos, inducción de parto, complicación de T. Parto, ápgar y volumen de sangrado. Resultados: El tipo de complicación puerperal más frecuente fue la Infección puerperal $61,19 \%$ seguida de Hemorragia puerperal $30,60 \%$. No hubo asociación estadística entre Embarazo a Término y complicación puerperal. De los factores estudiados el $\mathrm{N}^{\circ}$ CPN insuficientes tuvo 4,5 veces mayor probabilidad de presentar complicación, así como otros factores tales como la multiparidad, el sobrepeso, la anemia y la presencia de complicación durante el parto tuvieron mayor probabilidad de presentar complicación. Conclusiones: Se encontró que no existe asociación significativa entre la clasificación de embarazo en sus diferentes subclasificaciones y la presencia de complicaciones durante puerperio, en términos de desarrollar mayor complicación, generar un tipo en específico de complicación y en un manejo diferenciado entre uno u otra clasificación de E. a término.
\end{abstract}

Palabras claves: Embarazo a término, complicación puerperal, factor de riesgo. (Fuente: DeCS BIREME).

\section{OBSTETRIC COMPLICATIONS DURING THE POSTPARTUM PERIOD, IN RELATION TO THE NEW CLASSIFICATION OF PREGNANCY AT THE END OF THE ACOG, IN THE HOSPITAL OF VITARTE, YEAR 2018}

\begin{abstract}
Objective: Determine the association between puerperal complications and the classification of Pregnancy to Term of the ACOG, in the Hospital of Vitarte, year 2018. Materials and methods: The study carried out is analytical, retrospective casetype and control. The study population was made up of patients with intermediate postpartum period and who had some complication during this period. It was represented by 268 patients, with 134 cases and 134 controls with a ratio of 1:1. The information was collected from the Clinical Histories and these were: age, degree of instruction, parity, $\mathrm{N}^{\circ} \mathrm{CPN}, \mathrm{RPM}, \mathrm{BMI}$, hemoglobin, term pregnancy, type of delivery, duration of vaginal delivery, use of oxytocics, induction of labor, complication of J. labor, Apgar and volume of bleeding. Results: The most common type of puerperal complication was puerperal infection $61.19 \%$ and puerperal hemorrhage $30.60 \%$. There was no statistical association between Pregnancy to Term and puerperal complication. Of the factors studied, $\mathrm{N}^{\circ}$ insufficient CPN was 4.5 times more likely to present complication as well as other factors such as multiparity, overweight, anemia and the presence of complication during childbirth. Conclusions: It was found that there is no significant association between the classification of pregnancy in its different subclassifications and the presence of complications during puerperium. In terms of developing more complication, develop a specific type of complication and in a differentiated management between one or another classification of $\mathrm{E}$. to term.
\end{abstract}

Keywords: Full-term pregnancy, postpartum complication, risk factor (Source: MeSH NLM).

\section{INTRODUCCIÓN}

En las últimas décadas la Mortalidad Materna se ha convertido en una problemática muy importante a vencer por parte de todas las naciones del mundo, tanto así que la OMS nos menciona que cada minuto que pasa en alguna parte del mundo fallece una madre, dando datos incluso que por día hay más de 800 muertes maternas y menciona que en el año 2015 hubieron más de 300 000 muertes de este grupo poblacional producidas por complicaciones propias de la gestación, el parto y el puerperio. A pesar de estos datos desalentadores estadísticas a nivel Latinoamérica y el Caribe menciona una reducción de esta en un 40\% y a nivel nacional de un $43 \%$ pero no siendo suficiente para alcanzar el Objetivo del milenio ${ }^{1,2,3,4}$. 
También cabe recalcar que la muerte de las madres es un gran indicador de desarrollo de un país y si este indicador perdura y crece en el tiempo refleja el pobre interés que un Estado está dando para su nación y más sabiendo que esta situación es muy bien prevenible incluso evitable ${ }^{5}$. Además la literatura nos recalca a nivel Perú, las principales causas de fallecimiento materno, ocurren durante el puerperio ${ }^{6}$ y esto es producido por complicaciones que se darán en este periodo, siendo las más frecuentes de ocasionar esta fatalidad, las Hemorragias puerperales, otras como las Infecciones puerperales, las Hipertensiones Postparto y enfermedades Psiquiátricas puerperales ${ }^{7,8,9,10}$, y siendo la causa más frecuente de hemorragia, la atonía uterina $(82,4 \%)^{11}$.

\section{MATERIALES Y MÉTODOS}

Diseño y Población de estudio. El diseño del presente estudio es de tipo observacional, Retrospectivo, analítico de tipo casos y controles en pacientes con puerperio mediato y Embarazo a Término según la ACOG atendidas en el servicio de Gíneco-obstetricia del Hospital de Vitarte, entre enero a diciembre del año 2018. La muestra estuvo conformada por pacientes según la definición de casos y controles que se presenta a continuación: Los casos está representados por las pacientes mujeres con diagnóstico de cualquier complicación durante el puerperio y que tuvieron Embarazo a término según la ACOG y los controles, por pacientes mujeres sin diagnóstico de complicación durante el puerperio y que tuvieron Embarazo a término según la ACOG además se precisó una relación de controles por caso siendo esta de 1 , necesitándose 133 casos y 133 controles y así tener un ODDS RATIO de 4,2. Asumiendo que la tasa de expuestos de los que son grupo control será del 0.033 , usando como el factor de riesgo la Retención de Tejido Placentario, teniendo una prevalencia de 3,3\%.

El Departamento de Estadística nos proporcionó el listado total de pacientes que presentaron puerperio mediato del año 2018 , siendo estos 1948 , de las cuales 210 presentaron complicación durante puerperio, determinándose de forma aleatorizada 130 que tenían la definición de casos y de las 1738 pacientes restantes se obtuvo los otros 130 pacientes con la definición de controles.

\section{Técnicas y procedimientos de recolección de datos}

Se diseñó un formulario que incluyó las variables asociadas al tema de investigación, las cuales fueron analizadas, estudiadas y se presentan a continuación: edad, control prenatal, paridad, tipo de parto, grado de instrucción, RPM, IMC, duración de parto, hemoglobina, grado de sangrado, si presentaron complicación T. parto, el tipo de complicación de parto, si se usó oxitocina, si hubo inducción del parto, Ápgar del RN y embarazo a término. Esta ayuda que nos da este cuestionario fue potenciada dado que la mayoría fueron preguntas cerradas que ayudaron en la parte estadística. Toda la información que se pudo recolectar de las historias clínicas de las pacientes según la definición de casos y controles fueron digitalizadas en una hoja de cálculo de Excel, posteriormente se procedió a validar la información revisando por segunda vez cada ficha y así evitar el posible error de digitación u omisión de datos que puedan contribuir a obtener resultados no veraces.

Análisis de Datos. Consistió en identificar los factores que estén relacionados a complicaciones puerperales y estas a embarazo a término y además se les realizó las descripciones bivariadas respectivas a las que representaron naturaleza cualitativa y cuantitativa. También se usó resúmenes de frecuencia porcentuales y absolutas, además de medidas de comparación proporcional en función del chi cuadrado para las distintas variables categóricas y cuantitativas. Dentro de las pruebas inferenciales usadas fue el chi cuadrado; el valor decisivo para nosotros tomar alguna decisión estadística fue a través de establecer un nivel de significancia del 0.05 y nivel de confianza del $95 \%$, finalmente el procesamiento de los datos fue realizado en una hoja de cálculo Excel y el uso del software estadístico SPSS versión 25.

Consideraciones éticas. Debido a que este estudio tiene un método de recolección de datos de forma secundaria o indirecta, este no ocasionará ningún daño a la integridad de los pacientes. El único posible factor a tener en cuenta es evitar el reconocimiento directo o indirecto de mi unidad de análisis, por lo que se tratará de codificarlos de una forma bien establecida. Durante el presente estudio el investigador tendrá la responsabilidad de salvaguardar el completo anonimato de todos los sujetos de estudio. De tal forma que se tratará de cumplir la regulación de aprobación de protocolos de investigación del Hospital Vitarte, y este mismo será sometido a aprobación por parte del comité de investigación de dicho hospital.

\section{RESULTADOS}

El grupo estudiado estuvo conformada por 266 pacientes atendidas en el servicio de Gíneco-Obstetricia del Hospital de Vitarte (definición de la muestra), de las cuales 133 presentaron complicaciones puerperales (muestra de casos) y 133 no presentaron este tipo de complicaciones (muestra de controles). Del total de pacientes estudiadas, los factores maternos preparto que se describen a continuación, como Grupos etarios, en los cuales los comprendidos entre 20-34 años representó el $71,3 \%$,fueron los más frecuentes; según Grado de Instrucción la mayoría tuvieron secundaria completa con $73,1 \%$ y la minoría no tuvo educación con $2,2 \%$; en relación a la Paridad la mayoría de puérperas fueron Primíparas con $44,8 \%$, siguiéndoles las Multíparas con 41,8\%; las mujeres que presentaron CPN Suficientes fueron el $72,4 \%$ y las que tuvieron CPN Insuficientes fueron el 27,6\%; la mayoría no tuvo RPM con 93,3\% y las que sí tuvieron RPM representaron el 6,7\%; de acuerdo al IMC el Peso Normal representó el $82,8 \%$,seguido del Sobrepeso con $15,7 \%$; la 
mayoría tuvieron Hemoglobina Normal representando el $59,7 \%$ y Anemia Leve el $29,5 \%$; las puérperas tuvieron más frecuentemente Embarazo a Término Completo siendo el $75 \%$, seguido de Término Temprano el $18,3 \%$ y Embarazo a Término Tardío el 6,7\%(tabla1).

De todo el grupo estudiado las que tuvieron parto Vaginal representaron el $77,2 \%$ siendo las más frecuentes y las que tuvieron Cesárea fueron el $22,8 \%$,los que no usaron Oxitócicos representaron el $94 \%$ siendo los más frecuentes; la mayoría no tuvo Inducción del Parto representando el 94\%; en relación a la complicación del T. Parto la mayoría no la tuvo representando el $81 \%$;respecto a la duración de parto vaginal la mayoría tuvo una duración Normal representando el 79,8\%; según la duración de la Cesárea la mayoría tuvo una duración Normal representando el $90,2 \%$; la mayoría de los hijos de las puérperas tuvieron un Ápgar Adecuado representando el 99,6\%; en relación al Peso del recién nacido el Peso Normal representó el $96,6 \%$ finalmente el Grupo de sangrado más frecuente

Tabla 1. Factores v preparto de puérperas

\begin{tabular}{|c|c|c|}
\hline \multirow{2}{*}{ Variables } & \multicolumn{2}{|c|}{ Factores Maternos Preparto } \\
\hline & $\mathrm{n}$ & $\%$ \\
\hline \multicolumn{3}{|l|}{ Grupos etarios } \\
\hline 10 a 19 & 26 & $9,7 \%$ \\
\hline 20 a 34 & 191 & $71,3 \%$ \\
\hline$>=35$ & 51 & $19,0 \%$ \\
\hline \multicolumn{3}{|c|}{ Grado de Instrucción } \\
\hline Sin educación & 6 & $2,2 \%$ \\
\hline Primaria & 57 & $21,3 \%$ \\
\hline Secundaria & 196 & $73,1 \%$ \\
\hline Superior & 9 & $3,4 \%$ \\
\hline \multicolumn{3}{|l|}{ Paridad materna } \\
\hline nulípara & 36 & $13,4 \%$ \\
\hline primípara & 120 & $44,8 \%$ \\
\hline multípara & 112 & $41,8 \%$ \\
\hline \multicolumn{3}{|l|}{ Número de CPN } \\
\hline Insuficientes & 74 & $27,6 \%$ \\
\hline Suficientes & 194 & $72,4 \%$ \\
\hline \multicolumn{3}{|l|}{ RPM } \\
\hline si & 18 & $6,7 \%$ \\
\hline no & 250 & $93,3 \%$ \\
\hline \multicolumn{3}{|l|}{ IMC } \\
\hline Normopeso & 222 & $82,8 \%$ \\
\hline Sobrepeso & 42 & $15,7 \%$ \\
\hline Obesidad Tipo 1 & 4 & $1,5 \%$ \\
\hline Obesidad Tipo 2 & 0 & $0,0 \%$ \\
\hline Obesidad Mórbida & 0 & $0,0 \%$ \\
\hline \multicolumn{3}{|c|}{ Grados de Hemoglobina } \\
\hline Normal & 160 & $59,7 \%$ \\
\hline Anemia Leve & 79 & $29,5 \%$ \\
\hline Anemia Moderada & 29 & $10,8 \%$ \\
\hline Anemia Severa & 0 & $0,0 \%$ \\
\hline \multicolumn{3}{|c|}{ Embarazo a Término } \\
\hline Temprano & 49 & $18,3 \%$ \\
\hline Completo & 201 & $75,0 \%$ \\
\hline Tardío & 18 & $6,7 \%$ \\
\hline
\end{tabular}

Fuente: Datos recabados por el autor fueron $<=500$ cc y representó el 91,4\% seguido del grupo de $501-1000$ cc con $8,6 \%$. (Tabla 2 ).

Entre las complicaciones puerperales estudiadas, las más frecuentes fueron las Infecciosas con $61,19 \%$, seguida de las Hemorrágicas con $30,60 \%$, las Psiquiátricas un $4,48 \%$ y las Hipertensivas 3,73\%. (Gráfico 1).

Se muestra el análisis bivariado del Embarazo a Termino y la presencia de complicación puerperal, donde si bien en el E. Término Completo se presentó con mayor frecuencia, las complicaciones puerperales $(69,2 \%)$, este no presentó asociación estadística con la complicación puerperal, en el caso del a Término Temprano tampoco representó un aumento de la probabilidad de complicación puerperal ni significancia estadística (OR: 0,910(0,3962,089 ) /p: 0,669), de igual forma el E. Término Tardío no tuvo asociación estadística con la complicación puerperal (Tabla 3).

Se presenta el análisis bivariado de los factores maternos y perinatales en donde el tipo de parto que presentó más frecuencia de los que tuvieron complicación puerperal fue el Vaginal con $75,2 \%$, no mostrando significancia estadística; por otro lado el uso de oxitócicos aumentó 16 veces las probabilidades de presentar complicación puerperal además de significancia estadística 16,765(2,181$128,840) / p=0,00$; de igual forma la Inducción de parto es un factor que aumenta la probabilidad de generar complicación puerperal 16,765(2,181-128,840)/p=0,00; en relación a la complicación de parto también demostró aumentar 5 veces la probabilidad de realizar complicación durante el puerperio 5,467(2,604-11,477); de acuerdo a la duración de parto vaginal de los que presentaron complicación la mayoría tuvo una duración Normal 81,5\% y mostró significancia estadística; así mismo el Ápgar no tuvo asociación estadísticamente significativa; en relación al Grado de sangrado que presentó más frecuencia de los que tuvieron complicación puerperal fue $<=500 \mathrm{cc}$ con $91,7 \%$, no mostrando significancia estadística; con respecto al grupo etario, el grupo etario >=35 representó un factor protector contra la complicación puerperal; según Grado de Instrucción el grupo que tuvo secundaria completa fue el más frecuente con $70,7 \%$ aun así no presentó significancia estadística; el $\mathrm{N}^{\circ} \mathrm{CPN}$ insuficientes demostró aumentar 3 veces la probabilidad de presentar complicación puerperal; de los que presentaron complicación la mayoría no presentó RPM con $91 \%$, no siendo esta variable estadísticamente significativo; según la paridad materna, las multíparas y las nulíparas son factores que aumentan la probabilidad de presentar complicación puerperal según análisis bivariado; de acuerdo al IMC el sobrepeso representó un factor protector contra la complicación puerperal $0,329(0,164$ $0,658) / p=0,001$ al igual que la Anemia $0,338(0,204-0,563) /$ $\mathrm{p}=0,000$ (Tabla 4).

Podemos ver el análisis multivariado de los factores maternos y perinatales que resultaron tener significancia estadística en las tablas bivariadas de las cuales tuvieron 
asociación con las complicaciones puerperales las siguientes: La Multiparidad con OR: 2,292(1,224-4,294)/ $\mathrm{p}=0,010 ; \mathrm{N}^{\circ}$ CPN insuficientes OR: 4,517(2,13-9,219)/ $p=0,00$; el Sobrepeso con un OR: $3,937(1,614-9,602) /$ $p=0,03$; la Anemia OR: $4,318(2,273-8,203) / p=0,00$ y la Complicación de T. Parto OR: 6,986(2,810-17,370)/p=0,00 (Tabla 5).

Tabla 2. Factores perinatales de puérperas.

\begin{tabular}{|c|c|c|}
\hline \multirow[b]{2}{*}{ Variables } & \multicolumn{2}{|c|}{ Factores Perinatales } \\
\hline & $\mathrm{n}$ & $\%$ \\
\hline \multicolumn{3}{|l|}{ Tipo de Parto } \\
\hline vaginal & 207 & $77,2 \%$ \\
\hline cesárea & 61 & $22,8 \%$ \\
\hline \multicolumn{3}{|l|}{ Uso de Oxitócicos } \\
\hline si & 16 & $6,0 \%$ \\
\hline no & 252 & $94,0 \%$ \\
\hline \multicolumn{3}{|l|}{ Inducción de parto } \\
\hline si & 16 & $6,0 \%$ \\
\hline no & 252 & $94,0 \%$ \\
\hline \multicolumn{3}{|c|}{ Complicación T. Parto } \\
\hline si & 51 & $19,0 \%$ \\
\hline no & 217 & $81,0 \%$ \\
\hline \multicolumn{3}{|l|}{ Duración del Parto } \\
\hline Normal & 202 & $79,8 \%$ \\
\hline Prolongado & 8 & $3,2 \%$ \\
\hline Precipitado & 43 & $17,0 \%$ \\
\hline \multicolumn{3}{|c|}{ Ápgar del Recién Nacido } \\
\hline Adecuado & 267 & $99,6 \%$ \\
\hline Depresión Moderada & 1 & $0,4 \%$ \\
\hline Depresión Severa & 0 & $0,0 \%$ \\
\hline \multicolumn{3}{|l|}{ Grado del Sangrado } \\
\hline$<=500 c c$ & 245 & $91,4 \%$ \\
\hline $501-1000 c c$ & 23 & $8,6 \%$ \\
\hline$>1000 \mathrm{cc}$ & 0 & $0,0 \%$ \\
\hline
\end{tabular}

Fuente: Datos recabados por el autor

Tipo de Complicación Puerperal

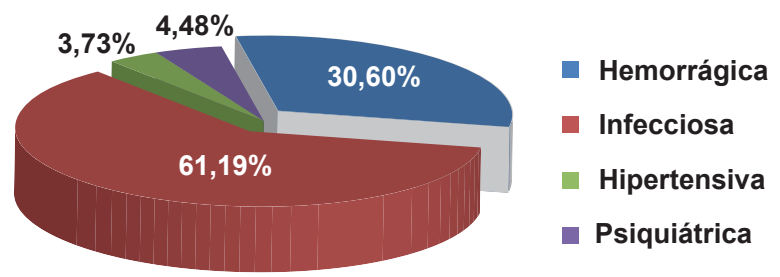

Gráfico 1. Tipo de Complicación durante el puerperio

\section{DISCUSIÓN}

Según el Tipo de complicación que predominó durante el puerperio, fueron las complicaciones Infecciosas 61,19\% las más frecuentes seguida de las Hemorrágicas 30,60\% cuyo resultado es muy similar al obtenido por Vargas Malena en un Hospital de Perú en el año 2017 12 , donde las Infecciosas representaron el $63,88 \%$ seguida de las
Hemorrágicas $36,12 \%$, según estos resultados se podría colegir como posible causa a la falta de equipamiento sanitario para el personal de salud, como también a la falta de responsabilidad de los padres a la asistencia de los CPN.

Según el análisis bivariado de la asociación entre Embarazo a Término y presencia de complicación; si bien en $\mathrm{E}$. Término Completo se presentó con mayor frecuencia las complicaciones puerperales $(69,2 \%)$, este no presentó asociación estadística con la complicación puerperal, en el caso del a Término Temprano tampoco representó un aumento de la probabilidad de complicación puerperal ni significancia estadística (OR: 0,910(0,3962,089) /p: 0,669), de igual forma el E. Término Tardío no tuvo asociación estadística con la complicación puerperal. Finalmente en el análisis multivariado ninguno de los que representa Embarazo a Término según la ACOG tuvo asociación significativa ni representó un aumento de la probabilidad de generar complicación puerperal.

Estos resultados podrían deberse a que si bien hay partos que se producen de forma temprana ( $E$. Término Temprano) y otros que se desarrollan muy tardíamente (E. Término Tardío) al parecer esto no influye mucho en desarrollar complicaciones puerperales talvez porque la anatomía materna está totalmente preparada para presentar el parto sin inconvenientes posteriores, en la clasificación a Término.

Tabla 3. Análisis Bivariado de Embarazo a Término y Presencia de Complicación Puerperal

\begin{tabular}{lrccccc}
\hline \multicolumn{6}{c}{ Presencia de Complicación } \\
\hline \multirow{2}{*}{$\begin{array}{l}\text { Embarazo } \\
\text { a Término }\end{array}$} & $\begin{array}{c}\text { Con } \\
\text { Complicación Complicación }\end{array}$ & p & $\begin{array}{c}\text { OR crudo e IC } \\
\text { AL 95\% }\end{array}$ \\
\cline { 2 - 8 } & $\mathbf{n}$ & $\%$ & $\mathbf{N}$ & $\%$ & \\
\hline Temprano & 32 & $24,1 \%$ & 17 & $12,6 \%$ & 0,058 & $2,23(1,16-4,27)$ \\
Tardío & 9 & $6,8 \%$ & 9 & $6,7 \%$ & $1,18(0,45-3,11)$ \\
Completo & 92 & $69,2 \%$ & 109 & $80,7 \%$ & & $*$ \\
\hline
\end{tabular}

Fuente: Datos recabados por el autor

Luego de haber analizado todas las Variables Maternas Preparto presentaron relación con las complicaciones las siguientes: CPN insuficientes, tuvo una asociación estadísticamente significativa para el desarrollo de complicación puerperal con un Odds Ratio de 4,517 y un valor de $p=0,00$ que a diferencia del estudio realizado por Huvin Karen ${ }^{13}$ donde menciona que si bien los CPN insuficientes le representa 1,06 veces mayor probabilidad de complicación, no tuvo significancia estadística con un $p=0,671$. Estos resultados de ambos trabajos puedan parecer muy lógicos ya que una madre si no previene todos los riesgos que pueda tener durante la gestación esto incrementaría la posibilidad que se presente complicaciones, no solo en el parto si no en el puerperio. Al igual que la Multiparidad en los resultados mostró evidencia que hay un valor de asociación significativa con la presencia de complicación puerperal con un Odds Ratio de 2,292 y un $p=0,010$. En contraste con el 
estudios realizado por Bustos Dany ${ }^{14}$ en el año 2016 donde nos dice que la Primiparidad puede incrementar hasta 5,76 la frecuencia de complicación puerperal $(\mathrm{OR}: 5,76)$ a su vez siendo este factor significativo $(p=0,013)$. Si bien ambos trabajos tuvieron resultados diferentes se puede concluir que la paridad en si representa un factor que aumenta la probabilidad de complicación durante el puerperio.

Además el presentar Anemia tuvo un resultado significativo de un $p=0,00$ y un OR: 4,318, representando este un factor que aumenta la probabilidad para desarrollar complicación puerperal a diferencia del estudio hecho por Parodi Jordán ${ }^{15}$ en el 2016 donde se concluyó que la presencia de Anemia no tuvo significancia estadística con un $\mathrm{p}=0,859$ para que se pueda producir complicación, mientras que en el trabajo realizado por Pereira Kevin ${ }^{16}$ en el 2018 el tener Anemia Leve incrementa hasta 4,34 veces la frecuencia para generar complicación puerperal tipo ITU. Teniendo estos resultados se puede decir que como sabemos, la anemia está asociada a disminución de las defensas y esto puede llevar a infecciones por colonización de bacterias y otras complicaciones.

Tabla 4: Análísis bivariado de los Factores Materno Perinatales

\begin{tabular}{|c|c|c|c|c|c|c|}
\hline \multirow{3}{*}{$\begin{array}{c}\text { Factores Maternos y } \\
\text { Perinatales }\end{array}$} & \multicolumn{6}{|c|}{ Presencia de Complicación } \\
\hline & \multicolumn{2}{|c|}{ Con Complicación } & \multicolumn{2}{|c|}{ Sin Complicación } & \multirow[t]{2}{*}{$\mathbf{p}$} & \multirow[t]{2}{*}{ OR crudo e IC AL $95 \%$} \\
\hline & $\mathbf{n}$ & $\%$ & $\mathbf{N}$ & $\%$ & & \\
\hline \multicolumn{7}{|l|}{ Tipo de Paro } \\
\hline Vaginal & 100 & $75,2 \%$ & 107 & $79,3 \%$ & 0.466 & $0,808(0,456-1,433)$ \\
\hline Cesárea & 33 & $24,8 \%$ & 28 & $20,7 \%$ & & * \\
\hline \multicolumn{7}{|l|}{ Uso de Oxitócicos } \\
\hline $\mathrm{Si}$ & 15 & $11,3 \%$ & 1 & $0,7 \%$ & 0,00 & $16,765(2,181-128,840)$ \\
\hline No & 118 & $88,7 \%$ & 134 & $99,3 \%$ & & * \\
\hline \multicolumn{7}{|l|}{ Inducción de parto } \\
\hline $\mathrm{Si}$ & 15 & $11,3 \%$ & 1 & $0,7 \%$ & 0,00 & $16,765(2,181-128,840)$ \\
\hline No & 118 & $88,7 \%$ & 134 & $99,3 \%$ & & * \\
\hline \multicolumn{7}{|l|}{ Complicación Parto } \\
\hline $\mathrm{Si}$ & 41 & $30,8 \%$ & 10 & $7,4 \%$ & 0,00 & $5,467(2,604-11,477)$ \\
\hline No & 92 & $69,2 \%$ & 125 & $92,6 \%$ & & * \\
\hline \multicolumn{7}{|l|}{ Duración del Parto Vaginal } \\
\hline Prolongado & 7 & $5,9 \%$ & 1 & $0,7 \%$ & & $7,58(0,92-62,71)$ \\
\hline Precipitado & 15 & $12,6 \%$ & 28 & $20,9 \%$ & & $0,58(0,29-1,15)$ \\
\hline Normal & 97 & $81,5 \%$ & 105 & $78,4 \%$ & 0,019 & * \\
\hline \multicolumn{7}{|l|}{ Ápgar del recién Nacido } \\
\hline Depresión Moderada & 1 & $0,7 \%$ & 1 & $0,7 \%$ & 0,421 & $1,32(0,26-3,17)$ \\
\hline Depresión Severa & 1 & $0,7 \%$ & 0 & $0,0 \%$ & & $0,94(0,02-1,78)$ \\
\hline Adecuado & 131 & $98,4 \%$ & 133 & $99,3 \%$ & & * \\
\hline \multicolumn{7}{|l|}{ Grado del Sangrado } \\
\hline $501-1000 \mathrm{cc}$ & 11 & $8,3 \%$ & 12 & $8,9 \%$ & & $0,92(0,39-2,17)$ \\
\hline$>1000 \mathrm{cc}$ & 0 & $0,0 \%$ & 0 & $0,0 \%$ & & 0,00 \\
\hline$<=500 \mathrm{cc}$ & 122 & $91,7 \%$ & 123 & $91,1 \%$ & 0,827 & * \\
\hline \multicolumn{7}{|l|}{ Grupos etarios } \\
\hline 10 a 19 & 15 & $11,3 \%$ & 11 & $8,1 \%$ & 0,021 & $1,22(0,53-2,78)$ \\
\hline$>=35$ & 17 & $12,8 \%$ & 34 & $25,2 \%$ & & $0,45(0,23-0,85)$ \\
\hline 20 a 34 & 101 & $75,9 \%$ & 90 & $66,7 \%$ & & * \\
\hline \multicolumn{7}{|l|}{ Grado de Instrucción } \\
\hline Sin educación & 3 & $2,3 \%$ & 3 & $2,2 \%$ & 0,166 & $3,5(0,37-32,97)$ \\
\hline Primaria & 34 & $25,6 \%$ & 23 & $17,0 \%$ & & $5,7(0,99-27,16)$ \\
\hline Secundaria & 94 & $70,7 \%$ & 102 & $75,6 \%$ & & $3,23(0,65-15,92)$ \\
\hline Superior & 2 & $1,5 \%$ & 7 & $5,2 \%$ & & * \\
\hline \multicolumn{7}{|l|}{ Número de CPN } \\
\hline Insuficientes & 51 & $38,3 \%$ & 23 & $17,0 \%$ & 0,00 & $3,228(1,818-5,732)$ \\
\hline Suficientes & 82 & $61,7 \%$ & 112 & $83,0 \%$ & & * \\
\hline \multicolumn{7}{|l|}{ RPM } \\
\hline si & 12 & $9,0 \%$ & 6 & $4,4 \%$ & 0,143 & $2,098(0,764-5,766)$ \\
\hline no & 121 & $91,0 \%$ & 129 & $95,6 \%$ & & * \\
\hline \multicolumn{7}{|l|}{ Paridad materna } \\
\hline nulípara & 30 & $22,6 \%$ & 6 & $4,4 \%$ & 0,00 & $9,63(3,71-25,02)$ \\
\hline multípara & 62 & $46,6 \%$ & 50 & $37,0 \%$ & & $2,39(1,41-4,06)$ \\
\hline primípara & 41 & $30,8 \%$ & 79 & $58,5 \%$ & & * \\
\hline IMC & & & & & & \\
\hline Sobrepeso & 33 & $24,6 \%$ & 13 & $9,7 \%$ & 0,001 & $0,329(0,164-0,658)$ \\
\hline Normopeso & 101 & $75,4 \%$ & 121 & $90,3 \%$ & & * \\
\hline Hemoglobina & & & & & & \\
\hline Anemia & 71 & $53,0 \%$ & 37 & $27,6 \%$ & 0,00 & $0,338(0,204-0,563)$ \\
\hline Normal & 63 & $47,0 \%$ & 97 & $72,4 \%$ & & * \\
\hline Embarazo a Término & & & & & & \\
\hline Temprano & 32 & $24,1 \%$ & 17 & $12,6 \%$ & 0,058 & $2,23(1,16-4,27)$ \\
\hline Tardío & 9 & $6,8 \%$ & 9 & $6,7 \%$ & & $1,18(0,45-3,11)$ \\
\hline Completo & 92 & $69,2 \%$ & 109 & $80,7 \%$ & & * \\
\hline
\end{tabular}

Fuente: Datos recabados por los autores; * Referencia 
Tabla 5. Análisis Multivariado de los Factores Maternos y perinatales según Presencia de Complicación Puerperal.

\begin{tabular}{|c|c|c|c|c|}
\hline Factores Maternos y perinatales & OR crudo e IC AL 95\% & $\mathrm{p}$ & OR ajustado e IC AL 95\% & $\mathrm{p}$ \\
\hline \multicolumn{5}{|l|}{ Uso de Oxitócicos } \\
\hline si & $16,765(2,181-128,840)$ & 0,00 & $4,052(0,003-4890,426)$ & 0,699 \\
\hline no & * & & * & \\
\hline \multicolumn{5}{|l|}{ Inducción de parto } \\
\hline si & $16,765(2,181-128,840)$ & 0,00 & $1,492(0,001-1865,621)$ & 0,912 \\
\hline no & * & & * & \\
\hline \multicolumn{5}{|l|}{ Complicación T.Parto } \\
\hline si & $5,467(2,604-11,477)$ & 0,00 & $6,986(2,810-17,370)$ & 0,00 \\
\hline no & * & & * & \\
\hline \multicolumn{5}{|l|}{ Número de CPN } \\
\hline Insuficientes & $3,228(1,818-5,732)$ & 0,00 & $4,517(2,13-9,219)$ & 0,00 \\
\hline Suficientes & * & & * & \\
\hline \multicolumn{5}{|l|}{ Paridad materna } \\
\hline Nulípara & $9,63(3,71-25,02)$ & & $7,673(2,470-23,841)$ & 0,824 \\
\hline multípara & $2,39(1,41-4,06)$ & & $2,292(1,224-4,294)$ & 0,010 \\
\hline Primípara & * & 0,00 & * & \\
\hline \multicolumn{5}{|l|}{ Grupos etarios } \\
\hline 10 a 19 & $1,22(0,53-2,78)$ & 0,021 & $1,31(0,55-2,94)$ & 0,721 \\
\hline$>=35$ & $0,45(0,23-0,85)$ & & $0,48(0,27-0,91)$ & \\
\hline 20 a 34 & * & & * & \\
\hline \multicolumn{5}{|l|}{ Embarazo a Término } \\
\hline Temprano & $2,23(1,16-4,27)$ & & $0,910(0,396-2,089)$ & 0,669 \\
\hline Tardío & $1,18(0,45-3,11)$ & & $0,525(0,124-2,212)$ & 0,824 \\
\hline Completo & * & 0,058 & * & \\
\hline \multicolumn{5}{|l|}{ IMC } \\
\hline Sobrepeso & $0,329(0,164-0,658)$ & 0,001 & $3,937(1,614-9,602)$ & 0,03 \\
\hline Normopeso & * & & * & \\
\hline \multicolumn{5}{|l|}{ Hemoglobina } \\
\hline Anemia & $0,338(0,204-0,563)$ & 0,00 & $4,318(2,273-8,203)$ & 0,00 \\
\hline Normal & * & & * & \\
\hline
\end{tabular}

Fuente: Datos recabados por los autores; * Referencia

Según el análisis bivariado de la asociación entre Embarazo a Término y presencia de complicación; si bien en $\mathrm{E}$. Término Completo se presentó con mayor frecuencia las complicaciones puerperales $(69,2 \%)$, este no presentó asociación estadística con la complicación puerperal, en el caso del a Término Temprano tampoco representó un aumento de la probabilidad de complicación puerperal ni significancia estadística (OR: 0,910(0,396-2,089) /p: 0,669), de igual forma el E. Término Tardío no tuvo asociación estadística con la complicación puerperal. Finalmente en el análisis multivariado ninguno de los que representa Embarazo a Término según la ACOG tuvo asociación significativa ni representó un aumento de la probabilidad de generar complicación puerperal.

Finalmente de todas las Variables Perinatales analizadas solo se obtuvo valor significativo a la presencia de Complicación durante el T. Parto, con un Odds Ratio de 6,98 y un valor de $p=0,00$, el cual comparado con el trabajo de Huvin Karen ${ }^{13}$ realizado en el año 2018 donde mencionan también un 2,25 veces probabilidad de presentar la complicación durante el puerperio además teniendo un $p=0,001$. Esto talvez puede producirse porque durante el parto es el momento idóneo donde cualquier error es repercutido en el puerperio a través de una complicación.

\section{DISCUSIÓN}

No se presentó asociación estadística significativa entre las pacientes con Embarazo a Término temprano y tardío en comparación con los de a término completo en relaciona a las complicaciones durante el puerperio.

De todas las complicaciones que se presentaron las más frecuentes fueron las Infecciones puerperales con $61,19 \%$ seguida de las Hemorragias puerperales $30,60 \%$.

De todas las Variables Maternas Preparto las que tuvieron asociación para desarrollar complicaciones puerperales son ${ }^{\circ} \mathrm{N}$ CPN insuficientes que tiene 4,5 veces mayor probabilidad de presentarlo, de igual forma la Multiparidad presentando 2,2 veces mayor probabilidad de generar estas complicaciones.

También hubo otros factores asociados como el Sobrepeso que presentó 3,9 veces mayor probabilidad de generar complicación puerperal así como la Anemia que tuvo 4,3 veces mayor probabilidad de generarlas.

Según el análisis de los Factores perinatales la presencia Complicación durante el T. Parto tiene 6,9 veces mayor probabilidad de presentar la complicación durante el puerperio. 
Financiamiento: Autofinanciado.

Conflicto de interés: Los autores declaran no tener algún conflicto de intereses

\section{REFERENCIAS BIBLIOGRÁFICAS}

1. Mortalidad materna [Internet]. [Citado 15 de marzo de 2020] Disponible en: https://www.who.int/es/news-room/fact-sheets/ detail/maternal-mortality.

2. del Carpio Ancaya L. Situación de la mortalidad materna en el Perú, 2000 - 2012. Rev perú med exp salud pública. Julio de 2013; 30(3):461-4

3. Mitchell C. OPS/OMS | Once países de América Latina y el Caribe registraron avances en la reducción de la mortalidad materna, según nuevo informe de la ONU [Internet]. Pan American Health Organization / World Health Organization. 2014 [citado 21 de diciembre de 2020]. Disponible en: https://www. paho.org/hq/index.php?option=com_co ntent \&view=article\&id=9552:2014-11-countries-latinamerica-caribbean-reduced-maternal-mortality-new-datashow\&ltemid=1926\&lang=es.

4. Guevara-Ríos E. Estado actual de la mortalidad materna en el Perú. Investigación Materno Perinatal. 17 de octubre de 2019 ; $5(2): 7-8$

5. Marrón-Peña M. Mortalidad materna: un enfoque histórico. Rev Mex Anest. 21 de febrero de 2018; 41(1):59-63.

6. Gil F. Situación Epidemiológica de la Muerte Materna en el Perú 2015. 2015; 25 (4):66 - 74.

7. Bustan RV. Complicaciones en Pacientes con Puerperio Inmediato Atendidas en el Servicio de Ginecología de Hospital Provincial General Docente Riobamba. Año 2008 [Internet] [Tesis]. [Riobamba-Ecuador]: Escuela Superior Politécnica De Chimborazo; 2010 [citado 15 de marzo de 2020]. Disponible en:http://dspace.espoch.edu.ec/handle/123456789/185

8. Naul L, Quiñones C. Complicaciones del puerperio inmediato en pacientes atendidas en el Servicio de Ginecología y Obstetricia del Hospital Vicente Corral Moscoso, Cuenca-2013. Revista Latinoamericana de Hipertensión. 2019; Vol. 14:205-6.

9. Martínez K, Brizuela E. Prevalencia de complicaciones puerperales y factores asociados en mujeres atendidas en el Hospital José Nieborowski de la Ciudad de Boaco durante el 2014 [Internet] [Tesis]. [Nicaragua]: Universidad Nacional
Autónoma de Nicaragua, Managua; 2015 [citado 15 de marzo de 2020]. Disponible en: http://repositorio.unan edu.ni/3637/.

10. Briones C, Medrano A, Paladines E, Chang A. Factores de riesgo y complicaciones más frecuentes del puerperio: estudio realizado en el hospital materno infantil Mariana de Jesús. Medicina. 2002; 8(2):110-3.

11. Ngwenya S. Postpartum hemorrhage: incidence, risk factors, and outcomes in a low-resource setting. Int J Womens Health. 2 de noviembre de 2016; 8:647-50.

12. Vargas M. Factores asociados a las complicaciones que se presentan durante el puerperio en un hospital general, Perú - 2016 [Internet] [Tesis]. [Lima - Perú]: Universidad Nacional Mayor de San Marcos; 2017 [citado 15 de marzo de 2020]. Disponible en: http://cybertesis.unmsm.edu.pe/handle/ cybertesis/6165.

13. Cruz HDL, Yessenia K. Factores asociados a complicaciones puerperales en mujeres peruanas: Subanálisis Endes 2016 [Internet] [Tesis]. [Lima - Perú]: Universidad federico Villarreal; 2018 [citado 15 de marzo de 2020].Disponibleen:http:// repositorio.unfv.edu.pe/handle/UNFV/1716.

14. Bustos D. Factores asociados a complicaciones en pacientes postcesareadas del Hospital Nacional Docente Madre Niño San Bartolomé, Lima 2014 [Internet] [Tesis]. [Lima - Perú]: Universidad Nacional Mayor de San Marcos; 2016 [citado 15 de marzo de2020]. Disponibleen:http://cybertesis.unmsm. edu.pe/handle/cybertesis/4698.

15. Parodi J. Complicaciones maternas durante el trabajo de parto y puerperio en gestantes con anemia atendidas en el Instituto Nacional Materno Perinatal, año 2015 [Internet] [Tesis]. [Lima - Perú]: Universidad nacional Mayor de San Marcos; 2016 [citado 15 de marzo de 2020]. Disponible en: http://cybertesis. unmsm.edu.pe/handle/cybertesis/4829.

16. Pereira C, Pereira K. Factores que se asocian a las complicaciones durante el puerperio en el Hospital María Auxiliadora (HAMA) Lima - Perú. [Internet] [Tesis]. [CuscoPerú]: Universidad Andina del Cusco; 2018 [citado 15 de marzo de 2020]. Disponible en: http://repositorio.uandina.edu. pe:8080/xmlui/handle/UAC/1446.

\section{Correspondencia:}

Eduardo Montenegro Rivera.

Dirección: AAHH. Villa Rosario Mz. B Lote 5 San Juan de Lurigancho

Correo: eduar_1079@hotmail.com

Teléfono: $917 \overline{3} 38424$ 